\title{
INACTIVATION OF THE MEK/ERK PATHWAY IN THE MYOCARDIUM DURING CARDIOPULMONARY BYPASS
}

Eugenio G. Araujo, DVMa,b

Cesario Bianchi, MD, $\mathrm{PhD}^{\mathrm{a}}$

Kaori Sato, MD

Renato Faro, $\mathrm{PhD}^{\mathrm{a}}$

X. Alec Li, PhD

Frank W. Sellke, MDa
Objectives: A general pro-inflammatory response after cardiopulmonary bypass (CPB) may involve changes in signal transduction and in part be responsible for arrhythmias and myocardial dysfunction after cardiac surgery. The MEK/ERK (mitogen-activated protein kinase kinase/extracellular regulated kinase) pathway is common to many stimuli and may play a pivotal role in morbidity associated with CPB. We investigated the changes in MEK/ERK pathway and related enzymes after CPB in pigs.

Methods: We examined ventricular and atrial tissue from pigs before $90 \mathrm{~min}$ utes of normothermic CPB and after 90 minutes of post-CPB perfusion. The activities and protein levels of kinases MEK1/2, ERK1/2, a cellular tyrosine kinase (c-Src), protein kinase B (Akt), and the protein levels of mitogen-activated protein kinase phosphatase (MKP-1) were studied by immunoblotting ventricular and atrial myocardium lysates and labeling sections with antibodies that recognize the activated forms of the kinases and the phosphatase. Control pigs were subjected to sternotomy and heparinization but not CPB.

Results: We found a consistent inactivation of MEK/ERK pathway in both ventricular and atrial myocardium with an increase in MKP-1, a negative regulator of ERK1/2. The activities and protein levels of c-Src and Akt were not significantly modified before or after CPB, suggesting a certain degree of specificity for the MEK/ERK pathway. Such changes were not observed in controls. The decrease of ERK1/2 and MEK1/2 phosphorylation 90 minutes after termination of CPB (as well as the increase of nuclear MKP-1 protein levels) was also apparent by confocal microscopy.

Conclusions: These results collectively reveal a prevalence of inhibitory mechanisms in the MEK/ERK signal transduction machinery in myocardium subjected to CPB. (J Thorac Cardiovasc Surg 2001;121:773-81)
C ardiopulmonary bypass (CPB) is an essential component of many cardiac operations. There is, however, significant morbidity associated with CPB includ-

From the Division of Cardiothoracic Surgery, Department of Surgery, ${ }^{a}$ Beth Israel Deaconess Medical Center, and Harvard Medical School, Boston, Mass, and the Department of Cellular Biology, ${ }^{\mathrm{b}}$ University of Brasilia, Brasilia, DF, Brazil.

Supported by grant RO1 HL46716 (F.W.S.) from the National Institutes of Health, National Heart, Lung, and Blood Institute.

E. G. Araujo is supported by a fellowship from Fundação Coordenação de Aperfeiçoamento de Pessoal de Nível Superior (CAPES), Brazil. R. Faro is supported by a fellowship from Fundação de Amparo a Pesquisa do Estado de São Paulo (FAPESP), Brazil.

Copyright (C) 2001 by The American Association for Thoracic Surgery

$0022-5223 / 2001 \$ 35.00+0 \quad \mathbf{1 2 / 1 / 1 1 2 9 3 3}$

doi: $10.1067 / \mathrm{mtc} .2001 .112933$ ing microvascular dysfunction (spasm and vasoplegia), ${ }^{1}$ increased endothelial permeability (edema), ${ }^{2}$ cardiac arrhythmias, and myocardial contractile dysfunction. ${ }^{3}$

Protein phosphorylation by kinases is critical in the regulation of enzymes and other cellular proteins. The 44-kDa extracellular regulated kinase (ERK1, p44 mitogen-activated protein [MAP] kinase) and its 42$\mathrm{kDa}$ isoform, ERK2 (p42 MAP kinase), have been determined to be involved in muscular contraction, 4,5 vascular tone, ${ }^{6}$ and myocardium survival and function. ${ }^{7}$ In addition, inhibition of MEK1/2 (mitogenactivated protein kinase kinase [MAPK kinase]), the kinase that phosphorylates ERK1/2, reduces edema induced by tetradecanoyl phorbol acetate in mice, ${ }^{8}$ pointing to a direct involvement of the MEK/ERK1/2 pathway with acute inflammation. ERK1/2 is activated by many different stimuli, contributing to cellular 


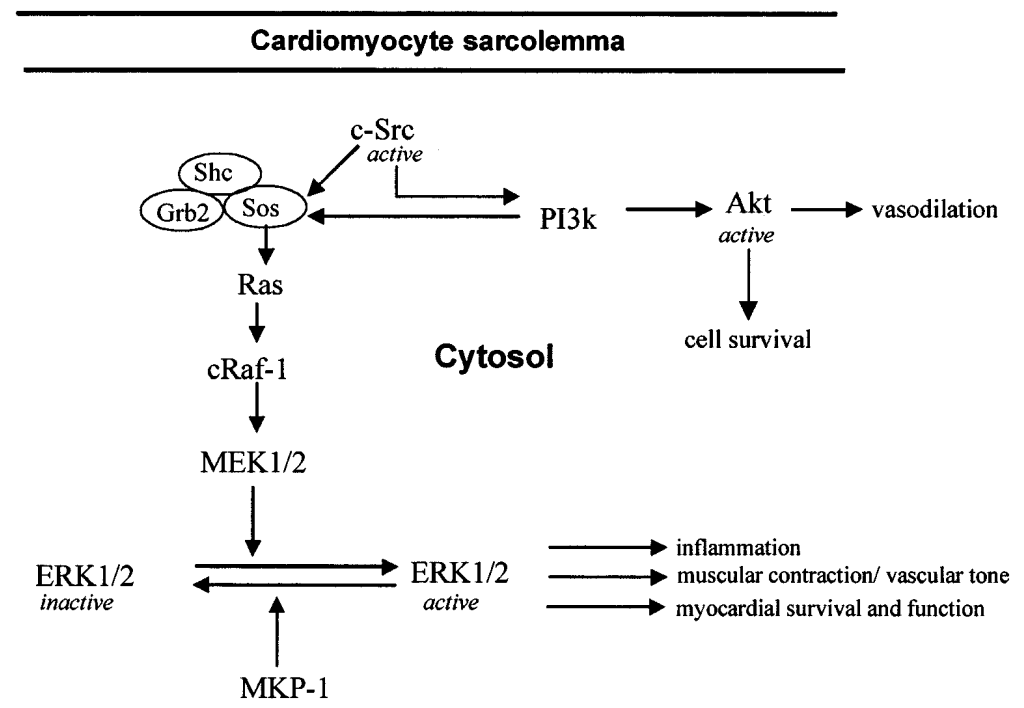

Fig 1. Simplified MEK/ERK pathway and its possible relation to c-Src and Akt (see text for details). ERK, Extracellular regulated kinase; $M E K$, mitogen-activated protein kinase kinase [MAPK kinase]; $M K P-1$, mitogenactivated protein kinase phosphatase; Akt, protein kinase B; $c$-Src, cellular tyrosine kinase pp60; PI3k, phosphatidylinositol 3-kinase.

proliferation and differentiation and cell cycle regulation and survival ${ }^{9}$ (Fig 1).

Deactivation of ERK1/2 can be mediated by MKP1/CL100, a dual specific phosphatase ${ }^{10}$ that dephosphorylates both serine and threonine residues (Fig 1). MKP-1 (MAP kinase phosphatase), a 39-kDa protein, is localized in the nucleus and can be rapidly induced by factors such as oxidative stress. ${ }^{11,12}$ It remains to be investigated whether MKP-1 is involved in the pathophysiologic mechanisms associated with CPB.

More recently, Akt (protein kinase B) (Fig 1), a 57$\mathrm{kDa}$ protein serine/threonine kinase, ${ }^{13-15}$ has been shown to improve survival of cardiomyocytes in culture deprived of serum, as well as to protect murine hearts against ischemia/reperfusion injuries in vivo. ${ }^{16}$ Thus, Akt could possibly play a role in myocardial protection during CPB. It has also been demonstrated in vivo that activation of Akt is associated with vasodilatation through a nitric oxide-dependent mechanism, ${ }^{17}$ suggesting that Akt could regulate the edema that follows CPB.

Activation of ERK1/2 may be dependent on another enzyme, c-Src, a tyrosine kinase (Fig 1). For example, c-Src plays a pivotal role in ERK1/2 activation by hydrogen peroxide in cultured cardiomyocytes. ${ }^{18}$ Furthermore, a c-Src inhibitor decreases Akt activity stimulated by ischemia/reperfusion in cultures of cardiomyocytes, ${ }^{19}$ pointing to a possible role of c-Src in post-CPB injury.
Given that the aforementioned signal transduction pathways are related to cardiac function and edema formation, we studied the protein levels and activity of selected enzymes in the pathway (Fig 1) in ventricular and atrial myocardium from pigs before and after $\mathrm{CPB}$. Using specific antibodies against the phosphorylated forms of Akt, ERK1/2, MEK1/2, activated c-Src, and MKP-1, we examined the state of their activation and protein steady state levels in myocardial tissue.

\section{Material and methods}

Animal preparation. Yorkshire pigs $(20-25 \mathrm{~kg})$ of either sex were premedicated with ketamine $(10 \mathrm{mg} / \mathrm{kg}$, intramuscularly) and anesthetized with $\alpha$-chloralose and urethane (60 $\mathrm{mg} / \mathrm{kg}$ and $300 \mathrm{mg} / \mathrm{kg}$ intravenously initially and then 15 $\mathrm{mg} / \mathrm{kg}$ and $60 \mathrm{mg} / \mathrm{kg}$ every 60 minutes as needed, respectively). Pigs were intubated and mechanically ventilated (Harvard Apparatus, Inc, S Natick, Mass). After the induction of anesthesia and tracheal intubation, a fluid-filled catheter was introduced into the femoral artery for the measurement of peripheral arterial pressure. After a sternotomy was performed, pigs were given heparin $(500 \mathrm{U} / \mathrm{kg})$ and cannulated via the distal ascending aorta and the right atrium through the purse-string sutures. Normothermic CPB was instituted with an oxygenator (Bentley, Bio-2; Baxter Healthcare Corp, Irvine, Calif) and standard roller pump. Hemodynamic parameters were maintained as described in detail previously. ${ }^{20}$ In brief, blood flow was maintained from 2.0 to $3.0 \mathrm{~L} \cdot \mathrm{min}^{-1}$ $\left(2.6-4.2 \mathrm{~L} \cdot \mathrm{min}^{-1} \cdot \mathrm{m}^{-2}\right)$ to assure a mean pressure of 40 to 70 $\mathrm{mm} \mathrm{Hg}$. Arterial blood gases were adjusted by ventilatory 
CPB

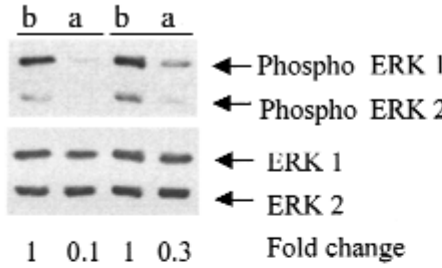

A

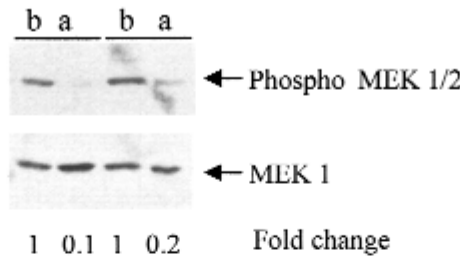

B

\section{Control}

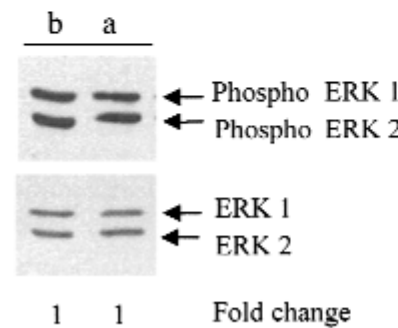

$\mathrm{D}$

Fig 2. Anti-phospho-ERK1/2 (A), anti-phospho-MEK1/2 (B), anti-MKP-1 (C), and respective control (D, E, and F) immunoblottings of total protein lysate from pig ventricle before $(b)$ and 90 minutes after $(a)$ post-CPB perfusion or 180 minutes after start of sham operations in controls as described in "Material and methods." ERK1/2 phosphorylation significantly decreases after 90 minutes of CPB and 90 minutes of post-CPB perfusion ( 0.39 -fold \pm 0.15 as compared with basal levels, $P=.006, \mathrm{n}=5)$, as well as MEK1/2 phosphorylation $(0.36$-fold \pm 0.22 as compared with basal levels, $P=.009, \mathrm{n}=5$ ), whereas MKP-1 levels increase perfusion (5.11-fold \pm 2.2 as compared with basal levels, $P=.03, \mathrm{n}=5$ ). No significant changes are observed in controls. The figure shows the result of 2 different experiments representative of 5 pigs studied (CPB) or 1 experiment representative of 4 controls. Ponseau $\mathrm{S}$ or total protein was used to ascertain proper protein fractionation and transfer.

rate and tidal volume to maintain $\mathrm{Po}_{2}$ more than $80 \mathrm{~mm} \mathrm{Hg}$, $\mathrm{PCO}_{2}$ more than 30 and less than $45 \mathrm{~mm} \mathrm{Hg}$, and $\mathrm{pH}$ between 7.30 and 7.45. After pigs were weaned from CPB, the mean post-CPB perfusion pressure was maintained between 40 and $70 \mathrm{~mm} \mathrm{Hg}$. Atrial $(\mathrm{n}=7)$ and ventricular $(\mathrm{n}=5)$ tissue samples were harvested before institution of 90 minutes of CPB and after 90 minutes of post-CPB perfusion. In control animals $(n=4)$, the same surgical procedure described above was used, except that after sternotomy, catheter placement, and heparinization, CPB was not instituted. Samples of atrial and ventricular myocardium were harvested after catheter placement (equivalent to before $\mathrm{CPB}$ ), after 90 minutes, and at the end of 180 minutes (equivalent to after 90 minutes of post-CPB perfusion).

All animals received humane care in compliance with the Beth Israel Deaconess Medical Center Animal Care and Use Committee and the National Research Council's "Guide for the Care and Use of Laboratory Animals," prepared by the Institute of Laboratory Animal Resources, National Research Council, and published by the National Academy Press, revised 1996.
Immunoblotting. Total lysate from atrial and ventricular tissue was obtained by homogenization (PowerGen 125 Homogenizer; Fischer, Pittsburg, Pa) for 30 seconds on ice in lysis buffer containing $1 \%$ NP-40 surfactant, $0.5 \%$ sodium deoxycholate, and $0.1 \%$ sodium dodecyl sulfate (SDS) and by centrifugation at $12,000 \mathrm{~g}$ for 10 minutes at $4{ }^{\circ} \mathrm{C}$ to separate solubilized from unsolubilized protein. The supernatant protein concentration was measured spectrophotometrically at a 595-nm wave length (DU640; Beckman Coulter, Inc, Fullerton, Calif) using a BCA protein assay kit (Pierce Chemical Company, Rockford, Ill). Total protein was fractionated on $10 \%$ SDS-polyacrylamide gel electrophresis and transferred to a polyvinylidene difluoride membrane (Immobilon-P; Millipore Corporation, Bedford, Mass) using a semi-dry transfer apparatus (Millipore). Membranes were stained with Ponseau S, ${ }^{21}$ digitized (see below), and then incubated with $5 \%$ nonfat dry milk in $50 \mathrm{mmol} / \mathrm{L}$ Tris- $\mathrm{HCl}$, $\mathrm{pH} 8.0,100 \mathrm{mmol} / \mathrm{L} \mathrm{NaCl}$, and $0.1 \%$ Tween 20 (TBST) buffer for 1 hour at room temperature to block nonspecific binding. Membranes were incubated with rabbit anti-phospho p44/42 MAPK (New England Biolabs, Beverly, Mass) 


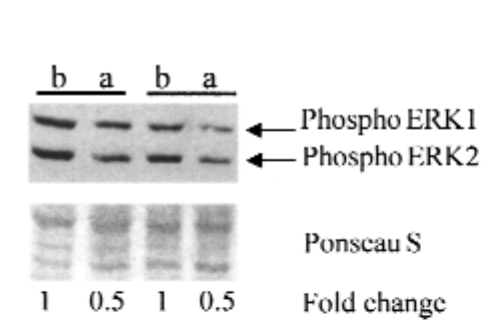

A

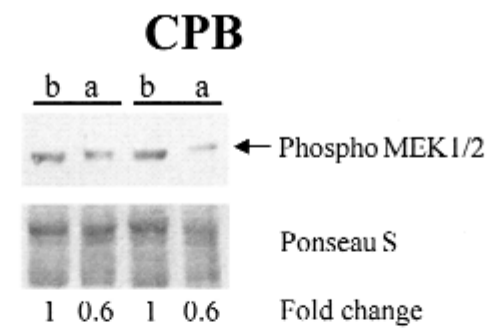

B

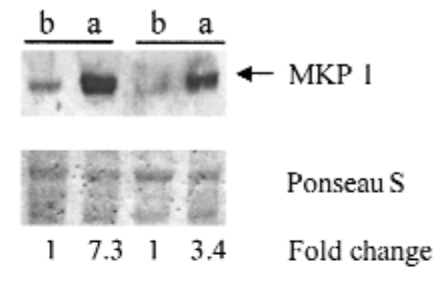

$\mathrm{C}$

\section{Control}



D

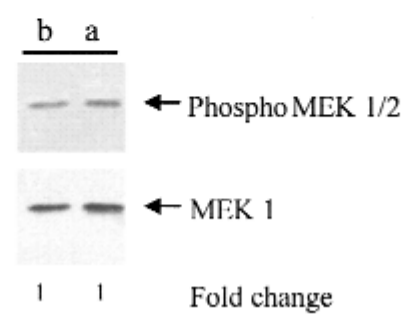

E



F

Fig 3. Anti-phospho-ERK1/2 (A), anti-phospho-MEK1/2 (B), anti-MKP-1 (C), and respective control (D, E, and F) immunoblottings of total protein lysate from pig atria before $(b)$ and 90 minutes after $(a)$ post-CPB perfusion or 180 minutes after start of sham operations in controls as described in "Material and methods." ERK1/2 phosphorylation significantly decreases after 90 minutes of CPB and 90 minutes of post-CPB perfusion tissue (0.47-fold \pm 0.10 as compared with basal levels, $P=.007, \mathrm{n}=7)$, as well as MEK1/2 phosphorylation $(0.49$-fold \pm 0.03 as compared with basal levels, $P=.008, \mathrm{n}=7$ ), whereas MKP-1 levels increased (4.35-fold \pm 1.15 as compared with basal levels, $P=.02, \mathrm{n}=4)$. The figure shows the result of 2 different experiments representative of 7 pigs studied $(\mathrm{CPB})$ or 1 experiment representative of 4 controls. Ponseau $\mathrm{S}$ or total protein was used to ascertain proper protein fractionation and transfer.

1:2500 (v/v) dilution in $2.5 \%$ nonfat dry milk, rabbit anti p44/42 MAPK (New England Biolabs) 1:2500 (v/v) dilution, rabbit anti-phospho MEK1/2 (New England Biolabs) 1:1000 (v/v) dilution, mouse anti-MEK-1 (Zymed Laboratories, San Francisco, Calif) 1:1000 (v/v) dilution, rabbit anti-phosphoAkt (New England Biolabs) 1:1000 (v/v) dilution, goat antiMKP-1 (V-15) (Santa Cruz Biotechnology, Santa Cruz, Calif) $1: 1000(\mathrm{v} / \mathrm{v})$ dilution, rabbit anti-c-Src (kindly provided by Dr Joan Brugges), and activated mouse anti-c-Src ${ }^{22}$ (generously provided by Dr Kawakatsu) 1/1000 (v/v) for 2 hours for immunoblotting. After being washed with TBST, the membranes were incubated for 1 hour in $2.5 \%$ nonfat dry milk in TBST containing 1:3000 diluted with the appropriated secondary antibody either a sheep anti-mouse (Amersham Pharmacia Biotech Inc, Piscataway, NJ), rabbit anti-goat immunoglobulin $\mathrm{G}$, or sheep anti-rabbit immunoglobulin $\mathrm{G}$ (Jackson Immunolabs, West Grove, Pa), at 1:5000 (v/v) dilution conjugated to horseradish peroxidase. Peroxidase activity was visualized by means of an enhanced chemiluminescence substrate system as previously described ${ }^{23}$ and exposed to x-ray films (Amersham, Arlington Heights, Ill).

Confocal microscopy. Frozen tissue samples were sectioned (5 $\mu \mathrm{m}$ thickness) in a cryostat (Tissue Tek; Miles Laboratories, Elkhart, Ind) and fixed for 20 minutes in $4 \%(\mathrm{w} / \mathrm{v})$ paraformaldehyde solution in phosphate-buffered saline solution (PBS), followed by permeabilization with $1 \%$ (w/v) SDS in PBS for 5 minutes. ${ }^{24}$ After being washed with PBS, sections were blocked overnight at $4^{\circ} \mathrm{C}$ with $1.5 \%$ bovine serum albumin against nonspecific binding. Different sections from the same tissues were then incubated for 1 hour at room temperature with rabbit anti-phospho p44/42 MAPK (New England Biolabs) 1:100 (v/v) dilution in 1.5\% (w/v) bovine serum albumin in PBS, rabbit anti-phospho MEK1/2 (New England Biolabs) 1:100 (v/v) dilution, or goat anti-MKP-1 (V-15) (Santa Cruz Biotechnology) 1:100 (v/v) dilution. The sections were washed 4 times in PBS and incubated for 30 minutes in 1.5\% bovine serum albumin in PBS containing a 1:300 dilution of the appropriated secondary antibody, a sheep anti-mouse (Sigma Chemical Co, St Louis, Mo), donkey anti-goat immunoglobulin $\mathrm{G}$ (Jackson Immunolabs), or sheep anti-mouse immunoglobulin $\mathrm{G}$ (Jackson Immunolabs), all conjugated with Cy3. The slides were then mounted with Vectashield (Vector Laboratories, Inc, Burlingame, Calif) and observed on a BioRad MRC-1024ES confocal microscope (Bio-Rad Microscience, Hercules, Calif).

Data acquisition and statistical analysis. Immunoblottings were analyzed after digitalization of x-ray films using a flat-bed scanner (ScanJet 4c; Hewlett-Packard Company, Palo Alto, 


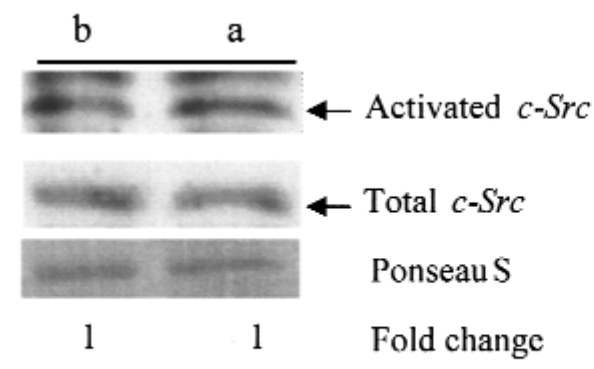

A

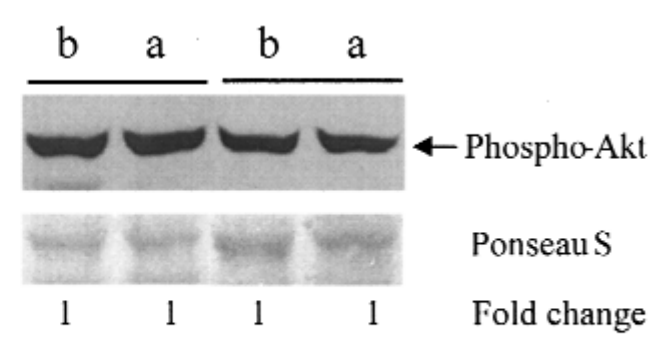

B

Fig 4. Immunoblotting of total protein lysate from pig atria before $(b)$ and 90 minutes after $(a)$ post-CPB perfusion as described in "Material and methods." A, The lane with total c-Src was probed with a c-Src antibody 327 . The lane with activated c-Src was probed with antibody that only recognizes c-Src in its active form (antibody 29). B, Akt phosphorylation was not significantly different between before and after post-CPB perfusion. The figure shows the results of 1 or 2 different experiments representative of 6 pigs studied. Ponseau $S$ was used to ascertain proper protein fractionation and transfer.

Calif) and NIH Image 1.62 software (National Institutes of Health, Bethesda, Md). Comparisons between samples were analyzed by 1-way analysis of variance followed by a 2-tailed $t$ test using Microsoft Excel software (Microsoft Corporation, Seattle, Wash). Values are expressed as mean fold change \pm standard deviation of the mean. Ponseau S staining was used to determine proper protein fractionation and equivalent loading. Only samples with similar protein fractionation and protein loading less than $20 \%$ differences were analyzed further. The optical density ratio of the bands to that of Ponseau $\mathrm{S}$ was used to correct for small uneven loading $(<20 \%)$.

\section{Results}

Phosphorylation levels of ERK1/2 decrease after CPB. The presence of an in vivo phosphorylation of ERK1/2 in basal levels was observed in both ventricular (Fig 2, $A$ and $D$ ) and atrial (Fig 3, $A$ and $D$ ) myocardium. Whereas levels of phosphorylated ERK1/2 decreased in ventricular myocardium (0.39fold \pm 0.15 as compared with basal levels, $P=.006$, $\mathrm{n}=5$ ) after 90 minutes of post-CPB perfusion (Fig 2, $A)$, levels did not decrease ( 1.07 -fold \pm 0.18 as compared with basal levels, $P>.2, \mathrm{n}=4)$ after 180 minutes in controls (Fig 2, D).

A similar picture was observed in atrial myocardium. After 90 minutes of post-CPB perfusion, a decrease in phosphorylated levels of ERK1/2 in atrial tissue ( 0.47 -fold \pm 0.10 as compared with basal levels, $P=.007, \mathrm{n}=7$ ) was consistently observed, as shown in Fig 3, A. However, protein levels of ERK1/2 were not modified after 90 minutes of post-CPB perfusion ( 0.96 -fold \pm 0.01 as compared with basal levels, $P=.06, \mathrm{n}=7$ ). In controls (Fig $3, D$ ), both phosphorylated (1.12-fold \pm 0.28 as compared with basal levels, $P=.15, \mathrm{n}=4)$ and total ERK1/2 $(1.02$-fold \pm
0.09 as compared with basal levels, $P>.2, \mathrm{n}=4$ ) levels did not significantly change after 180 minutes of sham operations not involving CPB.

Phosphorylation of MEK1/2 correlates with phosphorylation of ERK1/2. Since the steady state of ERK1/2 phosphorylation is altered after CPB, it is possible that similar changes would be observed in the phosphorylation steady state of MEK1/2, the upstream kinases that phosphorylate ERK1/2. As illustrated in Fig 2, $B$, phosphorylated levels of MEK1/2 significantly decreased in ventricular tissue $(0.36$-fold \pm 0.22 as compared with basal levels, $P=.009, \mathrm{n}=5$ ) after 90 minutes of $\mathrm{CPB}$ perfusion. However, they did not decrease (1.07-fold \pm 0.18 as compared with basal levels, $P=.10, \mathrm{n}=4)$ after a total of 180 minutes in controls (Fig 2, E). Such findings correlate well with ERK1/2 dephosphorylation.

As observed in ventricular myocardium, there was a prominent dephosphorylation of MEK1/2 in atrial myocardium (0.49-fold \pm 0.03 as compared with basal levels, $P=.008, \mathrm{n}=7$ ) (Fig 3, $B$ ) without significant changes in MEK1/2 protein levels (0.96-fold \pm 0.01 as compared with basal levels, $P>.2, \mathrm{n}=7$ ). In controls (Fig 3,E), neither phosphorylated MEK1/2 levels (1.12fold \pm 0.28 as compared with basal levels, $P>.2, \mathrm{n}=4$ ) nor total MEK-1 levels (1.08-fold \pm 0.1 as compared with basal levels, $P>.2, \mathrm{n}=4$ ) were significantly changed after 180 minutes of sham operations not involving CPB.

Induction of MKP-1 expression after CPB. A robust induction of MKP-1 immunoreactivity was observed in ventricular myocardium after 90 minutes of CPB and 90 minutes of post-CPB perfusion (5.11fold \pm 2.2 as compared with basal levels, $P=.03$, $\mathrm{n}=5$ ), as shown in Fig 2, $C$. At the same time point in 


\section{Phospho-ERK1/2}



A

\section{Before CPB}

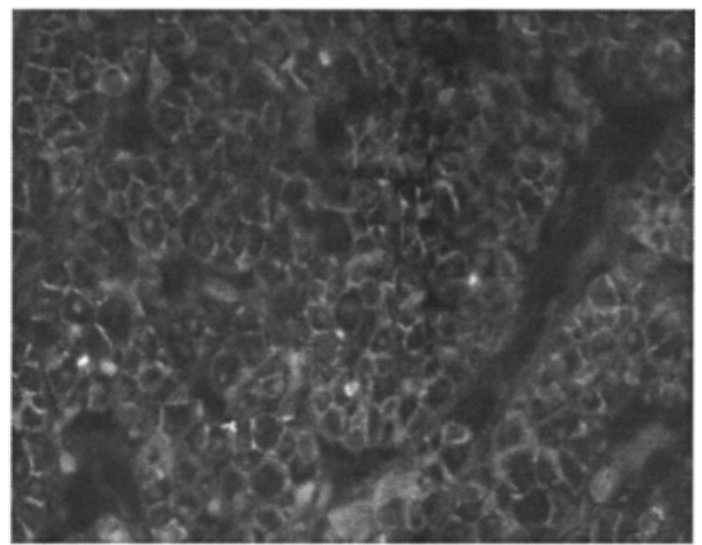

Post-CPB perfusion

\section{MKP-1}

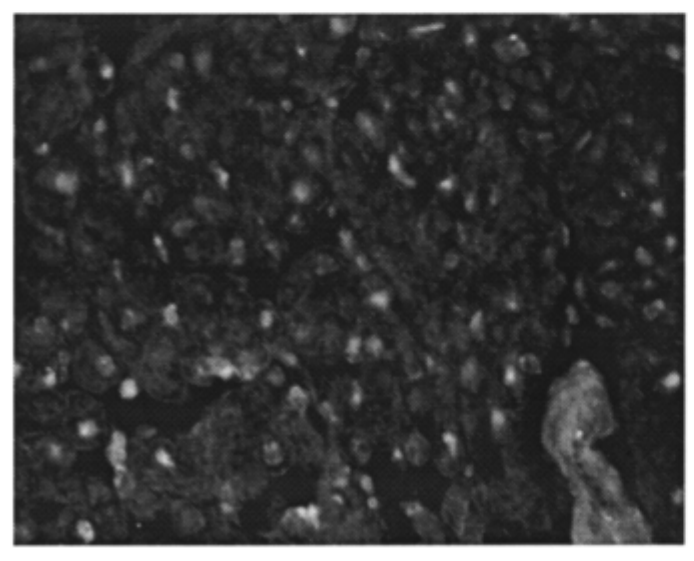

\section{Before CPB}

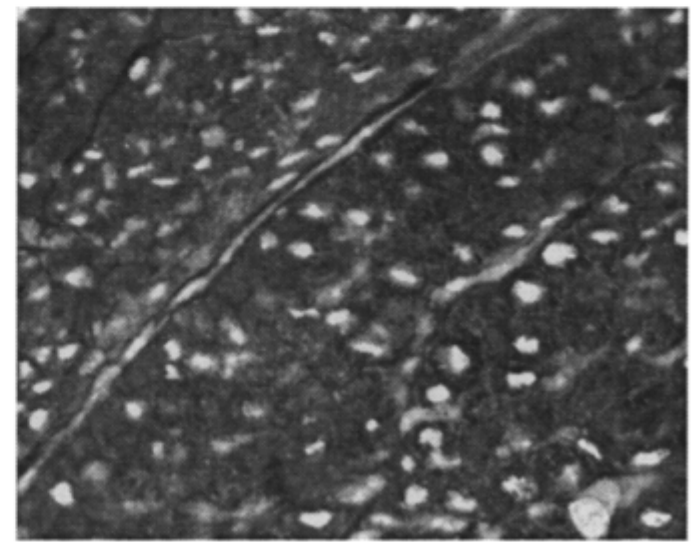

Post-CPB perfusion

Fig 5. A, Confocal microscopy images from pig atria before (left) and 90 minutes after (right) post-CPB perfusion as described in "Material and methods." Notice the intensity decrease of phospho-ERK1/2 signal post-CPB perfusion, confirming immunoblotting observations. Images were obtained from similar fields and same magnification and image adjustments. B, Confocal microscopy images from pig atria before (left) and 90 minutes after (right) post-CPB perfusion as described in "Material and methods." MKP-1 is induced at the end of post-CPB perfusion, localizing mainly in cardiomyocyte nuclei. Images were obtained from similar fields, magnifications, and adjustments.

controls (Fig 2,F), however, MKP-1 was not induced (1.07-fold \pm 0.13 as compared with basal levels, $P=.11, \mathrm{n}=4)$. It is interesting to note that MKP-1 can be detectable in samples before CPB, indicating basal expression in porcine myocardium.

Conspicuous induction of MKP-1 was also observed in atrial tissue 90 minutes after initiation of post-CPB perfusion (Fig 3, C) (4.35-fold \pm 1.15 as compared with basal levels, $P=.02, \mathrm{n}=4)$. In controls, however, MKP-1 expression (Fig 3,F) was not significantly altered (1.13-fold \pm 0.35 as compared with basal levels, $P=.08, \mathrm{n}=4)$.

Active forms of c-Src and phospho-Akt did not change after CPB. In the same atrial tissues as analyzed above, neither c-Src protein levels (1.01-fold \pm 0.01 as compared with basal levels, $P=.09, \mathrm{n}=6$ ) nor 
c-Src activity ( 0.99 -fold \pm 0.05 as compared with basal levels, $P>.2, \mathrm{n}=6$ ) were modified (Fig $4, A$ ). Similar results were obtained for activity of Akt $(0.98$-fold \pm 0.1 as compared with basal levels, $P=.17, \mathrm{n}=7$ ) and Akt protein levels (0.96-fold \pm 0.01 as compared with basal levels, $P>.2, \mathrm{n}=7$ ) (Fig 4, $B$ ).

In ventricular myocardium, Akt results were similar to those observed in atrial tissue. After 90 minutes of post-CPB perfusion (0.92-fold \pm 0.14 as compared with basal levels, $P>.2, \mathrm{n}=5)$ and in control pigs (1.02-fold \pm 0.07 as compared with basal levels, $P>$ $.2, n=4$ ), significant changes were not observed (data not shown).

Confocal microscopy of atrial tissue corroborate immunoblotting results. The decrease of ERK1/2 phosphorylation after 90 minutes of CPB and 90 minutes of post-CPB perfusion was also observed by confocal microscopy (Fig 5, A). Despite the difference in intensity, the phosphorylated form of ERK1/2 localized to the cardiomyocyte surfaces and nuclei before CPB and after 90 minutes of post-CPB perfusion.

Phosphorylated MEK1/2 was mainly observed in cardiomyocyte nuclei, with discrete cytoplasmic staining. Phosphorylated MEK1/2 was slightly decreased 90 minutes after CPB termination (data not shown).

MKP-1 immunostaining, almost exclusively observed on cardiomyocyte nuclei (Fig 5, B), confirmed the evident induction of expression of this dual phosphatase. Despite similar distribution at both time points, a more intense staining was observed after 90 minutes of post-CPB perfusion, and this correlated with immunoblotting results.

\section{Discussion}

The main findings of this study are that the levels of phosphorylated ERK1/2 were consistently lower than baseline after 90 minutes of normothermic CPB and 90 minutes of post-CPB perfusion. These decreases were accompanied by a decrease in MEK1/2 phosphorylation, indicating a decrease in upstream activation. MKP-1 protein levels were considerably increased, adding a downstream component to ERK1/2 inactivation. Because we were unable to detect any significant change in c-Src activity or phospho-Akt after CPB, a certain level of specificity to the MEK/ERK pathway is revealed. These results collectively point to a general decrease in the MEK/ERK signal transduction module after CPB.

The mechanisms regulating signal transduction have been studied mainly in cell culture models. Since cell cultures are kept "quiescent" before experimentation, inactivation of signal transduction from a basal level is frequently overlooked. In vivo, however, it appears that signal transduction pathways have a high basal level of activity. It is important to consider, as shown by immunoblotting and immunostaining, that basal levels of phosphorylated (active) ERK1/2 and MEK1/2, as well as MKP-1 expression, are detected in pig hearts. Because atrial and ventricular myocardium respond similarly to $\mathrm{CPB}$, it is suggested that atrial sampling (a more convenient approach in patients) may well reflect the changes occurring in ventricular myocardium.

It is interesting to note that the MEK/ERK pathway is inactivated by CPB. In fact, different models of ischemia/reperfusion using either cardiomyocyte cultures $^{7}$ or isolated hearts ${ }^{25-28}$ showed that ERK1/2 is activated after reperfusion. However, ERK1/2 phosphorylation shows a characteristic trend in these studies: rapid induction after initiation of reperfusion, reaching a peak and decreasing at the end of the reperfusion period. Consequently, it cannot be ruled out that during the 90 minutes of CPB and 90 minutes of post$\mathrm{CPB}$ perfusion, variations in the activity did not occur, nor can it be assumed that different results would not be obtained at later time points. Because in our protocol we harvested the samples after 90 minutes of postCPB perfusion, it is possible that ERK1/2 activation might have occurred before we collected our samples. In relation to our findings, it has been reported ${ }^{29}$ that ERK1/2 phosphorylation in human atrial tissue increases during cardioplegia; ERK1/2 activity was higher after ischemia and showed a further increase after reperfusion. The difference could be related to the fact that the present study does not use an ischemia/ reperfusion model, because cardioplegia was not used. In addition, in 6 of 8 patients from the study by Talmor and colleagues, ${ }^{29}$ the surgical procedure was shorter than our experimental design (ischemia, $44.3 \pm 7.7$ minutes; reperfusion, $36.3 \pm 15.7$ ), an important issue considering the possibility that the activation might not be sustained during the postoperative period.

Our data reveal not only that CPB induced the expression of MKP-1 in the heart, but also that MKP-1 expression is localized to the nuclei of the cardiomyocytes. This was not surprising considering that ERK1/2 dephosphorylation can be mediated by MKP1. ${ }^{10}$ For instance, angiotensin II, an activator of ERK1/2 in smooth muscle cells, ${ }^{30}$ induces MKP-1 protein synthesis within 1 hour of stimulation. More important, when protein synthesis is blocked and MKP-1 expression is inhibited, ERK1/2-stimulated phosphorylation is not reversed. ${ }^{31}$ Nevertheless, MKP1 cannot be solely responsible for ERK1/2 dephosphorylation in our CPB model, because MEK1/2 dephosphorylation clearly points to the involvement of upstream signals. Thus, CPB is likely to generate dif- 
ferent levels of ERK inactivation, one from upstream signals (MEK) and the other from parallel, inhibitory molecules (MKP-1).

To which degree the changes observed in the present study relate to the well-established morbidity associated with CPB is not clear at this time. However, our studies support an inactivation of MEK/ERK pathways that could contribute to myocardial depression observed after CPB. In fact, in vitro ${ }^{32}$ and ex vivo studies have revealed that PD98059, a MEK1/2 chemical inhibitor, prevented the increase in muscular contraction-induced ERK1/2 phosphorylation. Also, in rat aorta vascular smooth muscle cells subjected to mechanical stretch, ERK1/2 was phosphorylated to a peak of activity at 20 minutes, returning to basal levels after 30 minutes of stimulation. ${ }^{33}$ These observations suggest that, in our model, a certain degree of cardiomyocyte contraction dysfunction can be either a cause or effect of ERK1/2 dephosphorylation. Also, ERK1/2 inactivation can be a cause of the systemic and coronary vascular relaxation observed after CPB.

Another point to be considered is the role of ERK1/2 in preconditioning. In preconditioning, where the heart is subjected to short periods of ischemia and reperfusion before a sustained ischemia/reperfusion period, ${ }^{34}$ ERK activity increases up to $89 \%$ and translocates to the nucleus. ${ }^{35}$ Given that in our study ERK1/2 is dephosphorylated, it is likely that the hearts of the pigs subjected to CPB lacked the presumptive protective effect of preconditioning. Indeed, a recent pig model study shows that specific MEK1/2 inhibitors reduce ERK activity and may block the beneficial effects of preconditioning. ${ }^{36}$ This suggests that the inactivation of ERK during CPB may contribute to myocardial injury.

In vitro studies show that the addition of MEK1/2 inhibitor PD98059 to cardiomyocytes subjected to ischemia for 2 hours, followed by reoxygenation for 24 hours, induced apoptosis while inhibiting ERK phosphorylation completely. ${ }^{7}$ Consequently, the relationship between ERK dephosphorylation (also demonstrated in our data) and cardiomyocyte apoptosis may be clinically relevant in CPB. Likewise, the induction of MKP-1 that was observed after post-CPB perfusion should be considered detrimental to cardiomyocyte survival.

There is also ex vivo evidence that decreased ERK1/2 activity may be an important feature in cardiac dysfunction, because MEK1/2 inhibition (by pretreatment with PD98059) in isolated rat hearts subjected to ischemia and reperfusion reduced ERK activation by $71 \%$ and aggravated cardiac function injury. ${ }^{7}$ Thus, it appears that the ERK1/2 dephosphorylation in the myocardium after
post-CPB perfusion may have clinical significance with regard to preservation of cardiac function.

In conclusion, the MEK/ERK pathway is partially inactivated after 90 minutes of normothermic CPB followed by 90 minutes of post-CPB reperfusion in pigs. Further experiments need to determine the exact time course of ERK/MEK inactivation, as well as the role of the MEK/ERK pathway, in causing detrimental effects in the myocardium and the coronary and peripheral vasculature.

Received for publication June 7, 2000; revisions requested Aug 22, 2000; revisions received Oct 9, 2000; accepted for publication Nov 8, 2000.

Address for reprints: Frank W. Sellke, MD, Beth Israel Deaconess Medical Center, Division of Cardiothoracic Surgery, 110 Francis St, LMOB suite 2A, Boston MA 02215 (E-mail: fsellke@caregroup.harvard.edu).

\section{REFERENCES}

1. Sellke FW, Boyle EM Jr, Verrier ED. Endothelial cell injury in cardiovascular surgery: the pathophysiology of vasomotor dysfunction. Ann Thorac Surg 1996;62:1222-8.

2. Verrier ED, Morgan EN. Endothelial response to cardiopulmonary bypass surgery. Ann Thorac Surg 1998;66:S17-9; discussion S25-8.

3. Gomes WJ, Carvalho AC, Palma JH, Teles CA, Branco JN, Silas MG, et al. Vasoplegic syndrome after open heart surgery. J Cardiovasc Surg (Torino) 1998;39:619-23.

4. Aronson D, Violan MA, Dufresne SD, Zangen D, Fielding RA, Goodyear LJ. Exercise stimulates the mitogen-activated protein kinase pathway in human skeletal muscle. J Clin Invest 1997;99:1251-7.

5. Ryder JW, Fahlman R, Wallberg-Henriksson H, Alessi DR, Krook A, Zierath JR. Effect of contraction on mitogen-activated protein kinase signal transduction in skeletal musclel involvement of the mitogen- and stress-activated protein kinase 1. J Biol Chem 2000;275:1457-62.

6. Lagaud GJ, Lam E, Lui A, van Breemen C, Laher I. Nonspecific inhibition of myogenic tone by PD98059, a MEK1 inhibitor, in rat middle cerebral arteries. Biochem Biophys Res Commun 1999;257:523-7.

7. Yue TL, Wang C, Gu JL, Ma XL, Kumar S, Lee JC, et al. Inhibition of extracellular signal-regulated kinase enhances ischemia/reoxygenation-induced apoptosis in cultured cardiac myocytes and exaggerates reperfusion injury in isolated perfused heart. Circ Res 2000;86:692-9.

8. Jaffee BD, Manos EJ, Collins RJ, Czerniak PM, Favata MF, Magolda RL, et al. Inhibition of MAP kinase kinase (MEK) results in an anti-inflammatory response in vivo. Biochem Biophys Res Commun 2000;268:647-51.

9. Widmann C, Gibson S, Jarpe MB, Johnson GL. Mitogen-activated protein kinase: conservation of a three-kinase module from yeast to human. Physiol Rev 1999;79:143-80.

10. Keyse SM. The role of protein phosphatases in the regulation of mitogen and stress-activated protein kinases. Free Radic Res 1999;31:341-9. 
11. Keyse SM, Emslie EA. Oxidative stress and heat shock induce a human gene encoding a protein-tyrosine phosphatase. Nature 1992;359:644-7.

12. Lewis T, Groom LA, Sneddon AA, Smythe C, Keyse SM. XCL100, an inducible nuclear MAP kinase phosphatase from Xenopus laevis: its role in MAP kinase inactivation in differentiated cells and its expression during early development. J Cell Sci 1995;108:2885-96.

13. Coffer PJ, Woodgett JR. Molecular cloning and characterisation of a novel putative protein-serine kinase related to the cAMPdependent and protein kinase $\mathrm{C}$ families. Eur $\mathrm{J}$ Biochem 1991;201:475-81.

14. Jones PF, Jakubowicz T, Pitossi FJ, Maurer F, Hemmings BA. Molecular cloning and identification of a serine/threonine protein kinase of the second-messenger subfamily. Proc Natl Acad Sci U S A 1991;88:4171-5.

15. Bellacosa A, Testa JR, Staal SP, Tsichlis PN. A retroviral oncogene, akt, encoding a serine-threonine kinase containing an $\mathrm{SH}$ like region. Science 1991;254:274-7.

16. Fujio Y, Nguyen T, Wencker D, Kitsis RN, Walsh K. Akt promotes survival of cardiomyocytes in vitro and protects against ischemia-reperfusion injury in mouse heart. Circulation 2000;101:660-7.

17. Luo Z, Fujio Y, Kureishi Y, Rudic RD, Daumerie G, Fulton D, et al. Acute modulation of endothelial Akt/PKB activity alters nitric oxide-dependent vasomotor activity in vivo. J Clin Invest 2000;106:493-9.

18. Aikawa R, Komuro I, Yamazaki T, Zou Y, Kudoh S, Tanaka M, et al. Oxidative stress activates extracellular signal-regulated kinases through Src and Ras in cultured cardiac myocytes of neonatal rats. J Clin Invest 1997;100:1813-21.

19. Mockridge JW, Marber MS, Heads RJ. Activation of Akt during simulated ischemia/reperfusion in cardiac myocytes. Biochem Biophys Res Commun 2000;270:947-52.

20. Tofukuji M, Stahl GL, Agah A, Metais C, Simons M, Sellke FW. Anti-c5a monoclonal antibody reduces cardiopulmonary bypass and cardioplegia-induced coronary endothelial dysfunction. J Thorac Cardiovasc Surg 1998;116:1060-8.

21. Hassan J, Feighery C, Whelan A. Staining of molecular weight markers on nitrocellulose using Ponseau S [letter]. J Clin Lab Immunol 1987;24:104.

22. Kawakatsu H, Sakai T, Takagaki Y, Shinoda Y, Saito M, Owada $\mathrm{MK}$, et al. A new monoclonal antibody which selectively recognizes the active form of Src tyrosine kinase. J Biol Chem 1996;271:5680-5.

23. Thorpe GH, Kricka LJ. Enhanced chemiluminescent reactions catalyzed by horseradish peroxidase. Methods Enzymol 1986;133:331-53.
24. Wilson DM, Bianchi C. Improved immunodetection of nuclear antigens after sodium dodecyl sulfate treatment of formaldehydefixed cells. J Histochem Cytochem 1999;47:1095-100.

25. Bogoyevitch MA, Gillespie-Brown J, Ketterman AJ, Fuller SJ, Ben-Levy R, Ashworth A, et al. Stimulation of the stress-activated mitogen-activated protein kinase subfamilies in perfused heart: p38/RK mitogen-activated protein kinases and c-Jun N-terminal kinases are activated by ischemia/reperfusion. Circ Res 1996;79:162-73.

26. Mizukami Y, Yoshida K. Mitogen-activated protein kinase translocates to the nucleus during ischaemia and is activated during reperfusion. Biochem J 1997;323:785-90.

27. Takeishi Y, Abe J, Lee JD, Kawakatsu H, Walsh RA, Berk BC. Differential regulation of $\mathrm{p} 90$ ribosomal S6 kinase and big mitogen-activated protein kinase 1 by ischemia/reperfusion and oxidative stress in perfused guinea pig hearts. Circ Res 1999;85:1164-72.

28. Omura T, Yoshiyama M, Shimada T, Shimizu N, Kim S, Iwao H, et al. Activation of mitogen-activated protein kinases in in vivo ischemia/reperfused myocardium in rats. $\mathrm{J}$ Mol Cell Cardiol 1999;31:1269-79.

29. Talmor D, Applebaum A, Rudich A, Shapira Y, Tirosh A. Activation of mitogen-activated protein kinases in human heart during cardiopulmonary bypass. Circ Res 2000;86:1004-7.

30. Duff JL, Marrero MB, Paxton WG, Charles CH, Lau LF, Bernstein KE, et al. Angiotensin II induces 3CH134, a proteintyrosine phosphatase, in vascular smooth muscle cells. J Biol Chem 1993;268:26037-40.

31. Andersson MB, Ketterman AJ, Bogoyevitch MA. Differential regulation of parallel mitogen-activated protein kinases in cardiac myocytes revealed by phosphatase inhibition. Biochem Biophys Res Commun 1998;251:328-33.

32. Hayashi T, Hirshman MF, Dufresne SD, Goodyear LJ. Skeletal muscle contractile activity in vitro stimulates mitogen-activated protein kinase signaling. Am J Physiol 1999;277:C701-7.

33. Numaguchi K, Eguchi S, Yamakawa T, Motley ED, Inagami T. Mechanotransduction of rat aortic vascular smooth muscle cells requires RhoA and intact actin filaments. Circ Res 1999;85:5-11.

34. Schott RJ, Rohmann S, Braun ER, Schaper W. Ischemic preconditioning reduces infarct size in swine myocardium. Circ Res 1990;66:1133-42.

35. Ping $\mathrm{P}$, Zhang J, Huang S, Cao X, Tang XL, Li RC, et al. PKCdependent activation of p46/p54 JNKs during ischemic preconditioning in conscious rabbits. Am J Physiol 1999;277:H1771-85.

36. Strohm C, Barancik T, Bruhl ML, Kilian SA, Schaper W. Inhibition of the ER-kinase cascade by PD98059 and UO126 counteracts ischemic preconditioning in pig myocardium. J Cardiovasc Pharmacol 2000;36:218-29. 